\title{
Hiperinsulinismo congénito: caracterización de pacientes atendidos en un hospital universitario pediátrico en Colombia
}

\author{
Paola V. Pedraza-Flechas ${ }^{1,2,3,4}$, Rocío P. Pereira-Ospina ${ }^{1,2 *}$, Juan S. Molinares-Mejía², \\ José M. Suescún-Vargas ${ }^{1,2,3,4}$ y Javier Y. Pinzón-Salamanca ${ }^{1,2,3,4,5}$ \\ ${ }^{1}$ Instituto Roosevelt; ${ }^{2}$ Universidades del Rosario; ${ }^{3}$ Universidad de los Andes; ${ }^{4}$ Universidad de La Sabana; ${ }^{5}$ Universidad Militar Nueva Granada. \\ Bogotá, Colombia
}

\begin{abstract}
Resumen
Introducción: El hiperinsulinismo congénito es una enfermedad del metabolismo de la glucosa, fundamental en la endocrinología pediátrica, ya que se refiere a la producción de mayor cantidad de insulina de la necesaria según la glucemia, lo cual produce hipoglucemias graves persistentes. Esta alteración puede tener importantes secuelas neurológicas debido al daño irreversible que se produce en la neurona por la exposición a la hipoglucemia por cortos periodos de tiempo. Su diagnóstico no es sencillo y requiere un alto índice de sospecha. El tratamiento se establece de manera secuencial, en varias etapas, observando la respuesta a cada uno de los posibles medicamentos empleados. En caso de que falle el manejo farmacológico, se requieren procedimientos quirúrgicos. Serie de casos: Se presentan siete casos de hiperinsulinismo congénito que fueron diagnosticados en los últimos 7 años en el Instituto Roosevelt en Bogotá, Colombia. En este país, el radiotrazador empleado usualmente durante la tomografía por emisión de positrones (PET/TC) no se encuentra disponible. Sin embargo, en uno de los casos descritos fue posible emplear otro radiotrazador alternativo que permitió un adecuado diagnóstico y un tratamiento quirúrgico exitoso. Conclusiones: El hiperinsulinismo congénito es una condición clínica compleja que amerita un correcto diagnóstico y un apropiado manejo, con el objetivo de evitar el daño neurológico que producen las hipoglucemias persistentes. Es posible emplear PET/TC con un radiotrazador adecuado para realizar un diagnóstico oportuno y proporcionar la mejor opción terapéutica disponible.
\end{abstract}

Palabras clave: Hiperinsulinismo. Hipoglucemia. Insulina. Diazóxido. Octreotida.

\section{Congenital hyperinsulinism: characterization of patients treated in a pediatric university hospital in Colombia}

\begin{abstract}
Background: Congenital hyperinsulinism is a disease of the glucose metabolism, relevant in pediatric endocrinology because of the elevated production of insulin according to blood glucose level, which leads to persistent severe hypoglycemia. This condition can produce important neurological sequelae in the patient due to the irreversible damage that occurs in the neuron caused by the exposure to hypoglycemia for short periods of time. Congenital hyperinsulinism diagnosis is not simple
\end{abstract}

Disponible en internet: 23-03-2020 Bol Med Hosp Infant Mex. 2020;77(2):94-99 www.bmhim.com (http://creativecommons.org/licenses/by-nc-nd/4.0/). 
and it requires a high index of suspicion. The treatment should be established sequentially, in several steps, noticing the response to each possible medication used. If the pharmacological management fails, surgical procedures are required occasionally. Case series report: Seven cases of congenital hyperinsulinism diagnosed in the last seven years at the Instituto Roosevelt in Bogotá, Colombia are presented. In this country, the radiotracer used internationally during positron emission tomography/computed tomography (PET/CT) is not available. However, was possible to use an alternative radiotracer in one of the cases, which led to an adequate diagnosis and a successful surgical treatment. Conclusions: Congenital hyperinsulinism is a complex clinical condition, which requires proper diagnosis and treatment, with the aim of avoiding any neurological damage caused by persistent hypoglycemia. PET/CT can be used with an appropriate radiotracer for a timely diagnosis and to provide the best available therapeutic option.

Key words: Hyperinsulinism. Hypoglycemia. Insulin. Diazoxide. Octreotide.

\section{Introducción}

El hiperinsulinismo congénito $(\mathrm{HC})$ es una enfermedad genética en la que existe una secreción inapropiada de insulina, por parte de las células beta pancreáticas, que produce hipoglucemia persistente. Se caracteriza por la ausencia de cuerpos cetónicos y ácidos grasos en sangre y orina, con una adecuada respuesta al glucagón exógeno $0^{1,2}$.

La incidencia de HC es de 1 por cada 40,000-50,000 nacidos vivos en la población general. En las relaciones consanguíneas, la incidencia es mucho mayor (1 por cada 2,500 nacidos vivos). El HC es la principal causa de hipoglucemia persistente y recurrente en el primer año de edad ${ }^{2-4}$, lo cual provoca un alto riesgo de desarrollar convulsiones y daño cerebral permanente si no es tratada ${ }^{1}$. La hipoglucemia persistente se presenta en el $25-50 \%$ de los pacientes con $\mathrm{HC}$ debido al retraso en el diagnóstico y al tratamiento inadecuado.

La presentación clínica y la gravedad de la $\mathrm{HC}$ varían según la edad ${ }^{1-3}$ : durante el periodo gestacional se puede manifestar con macrosomía fetal, y durante el periodo neonatal, con hipoglucemia sintomática y convulsiones ${ }^{1,2,4}$. En esta enfermedad se presentan mutaciones genéticas en las regiones codificantes de las subunidades de los canales de potasio dependientes de trifosfato de adenosina (ATP) de las células beta pancreáticas. Estos canales son el receptor de sulfonilurea SUR 1 (codificado por el gen ABCC8) y el canal rectificador de entrada de potasio o poro de potasio KIR 6.2 (codificado por el gen KCNJ11) ${ }^{3,5}$. El cierre de los canales de ATP dependientes de potasio produce la despolarización de la membrana plasmática, la activación de los canales de calcio dependientes de voltaje, la elevación del calcio citosólico y la liberación de insulina a la circulación. Otras vías de amplificación pueden incrementar la liberación de insulina al elevarse el calcio citosólico, incluyendo moléculas derivadas de la mitocondria, inervación parasimpática y receptores
Tabla 1. Genes implicados en el desarrollo de hiperinsulinismo congénito ${ }^{1,4-8}$

\begin{tabular}{|l|l|}
\hline Gen & Función \\
\hline ABCC8 & $\begin{array}{l}\text { Relacionado con la producción de canales de potasio } \\
\text { dependientes de ATP de células beta pancreáticas }\end{array}$ \\
\hline KCNJ11 & $\begin{array}{l}\text { Relacionado con la producción de canales de potasio } \\
\text { dependientes de ATP de células beta pancreáticas }\end{array}$ \\
\hline GLUD1 & Codifica la enzima glutamato deshidrogenasa \\
\hline GCK & Codifica la enzima glucocinasa \\
\hline HADHSC & $\begin{array}{l}\text { Codifica la enzima } \\
\text { L-3-hidroxiacil-CoA-deshidrogenasa }\end{array}$ \\
\hline SLC16A1 & Codifica el transportador tipo 1 de monocarboxilato \\
\hline HNF4 $\alpha$ & Codifica el factor nuclear hepatocítico 4 alfa \\
\hline HNF1 $\alpha$ & Codifica el factor nuclear hepatocítico 1 alfa \\
\hline UCP2 & $\begin{array}{l}\text { Codifica la proteína mitocondrial de } \\
\text { desacoplamiento tipo } 2\end{array}$ \\
\hline INSR & Gen codificante para el receptor de la insulina \\
\hline MCT-1 & Trasportador de monocarboxilato 1 \\
\hline
\end{tabular}

para incretinas intestinales, especialmente el receptor de péptido similar al glucagón (GLP-1).

Las mutaciones inactivantes en los canales de potasio son la forma más común y grave de HC (Tabla 1) 1,4-10. Otra causa menos frecuente es la presencia de mutaciones en genes codificantes de metabolitos intermediarios y de moléculas importantes dentro de la cascada de señalización intracelular (por ejemplo, ATP y difosfato de adenosina). Existen numerosos síndromes que se relacionan con esta condición, como el síndrome de Beckwith-Wiedemann, el síndrome de Perlman, el síndrome de Kabuki, el síndrome de Sotos, trastornos congénitos de la glucosilación tipo 1a o 1b, y el síndrome de Usher tipo $1 \mathrm{c}^{1,5}$.

La sospecha clínica de HC comienza al evidenciar hipoglucemia que requiere un aporte de glucosa 
intravenosa con altos requerimientos de flujo metabólico por más de 7 días. El diagnóstico diferencial incluye defectos en la producción de hormonas contrarreguladoras (como hormona de crecimiento y cortisol), en la oxidación de ácidos grasos mitocondriales, en la gluconeogénesis y en la glucogenólisis, entre otros.

El HC se puede clasificar por su duración (permanente o transitorio) o por su comportamiento histológico (focal o difuso) $)^{1,3,5}$. El HC transitorio suele relacionarse con embarazos de madres con diabetes (gestacional 0 dependiente de insulina), pacientes con síndrome de Beckwith-Weidemann, retraso en el crecimiento intrauterino y asfixia perinatal ${ }^{3}$. En el HC difuso se presenta daño de las células beta pancreáticas, mientras que el focal se caracteriza por hiperplasia adenomatosa productora de insulina (localizada en la cabeza del páncreas en el $30 \%$ de los casos) $)^{6,7}$.

Se recomiendan pruebas moleculares para evaluar la presencia de mutaciones en los genes ABCC8 y KCNJ11, dado que estos análisis ayudan a la diferenciación entre las formas focales y las difusas.

Los criterios diagnósticos para el $\mathrm{HC}$ son los siguientes ${ }^{1,2,4,6}$ :

- Hipoglucemia (< $45 \mathrm{mg} / \mathrm{dl})$ con valores de insulina no suprimidos (> $1 \mathrm{mU} / \mathrm{l})$

- Respuesta favorable al glucagón (aumento de más de $30 \mathrm{mg} / \mathrm{dl}$ de glucosa ante la administración de 0.5-1 mg de glucagón) $)^{2,8}$

- Cuerpos cetónicos negativos en orina y plasma

- Ácidos grasos libres en plasma, en valores normales o bajos ${ }^{8}$

- Dependencia prolongada de tratamiento preventivo de hipoglucemia durante los primeros meses/años de vida (flujo metabólico $>10 \mathrm{mg} / \mathrm{kg} / \mathrm{min}$ en neonatos)

- Insulina detectable durante la hipoglucemia con cifras elevadas de péptido $C$

\section{Casos clínicos}

Se realizó un análisis retrospectivo de los pacientes con diagnóstico de HC en los últimos 7 años (2012-2018) en el Departamento de Educación e Investigación del Instituto Roosevelt; se analizaron tres pacientes de sexo masculino y cuatro pacientes de sexo femenino (Tabla 2).

Se incluyó un paciente pretérmino. Cuatro nacieron por cesárea y tres por parto vaginal. El promedio del peso de los neonatos fue de $3.838 \mathrm{~kg}$. En dos casos, el APGAR (prueba de apariencia, pulso, gesticulación, actividad y respiración) al primer minuto fue $<7$. Dos pacientes de sexo masculino tenían antecedentes de diabetes en la familia y un paciente de sexo femenino

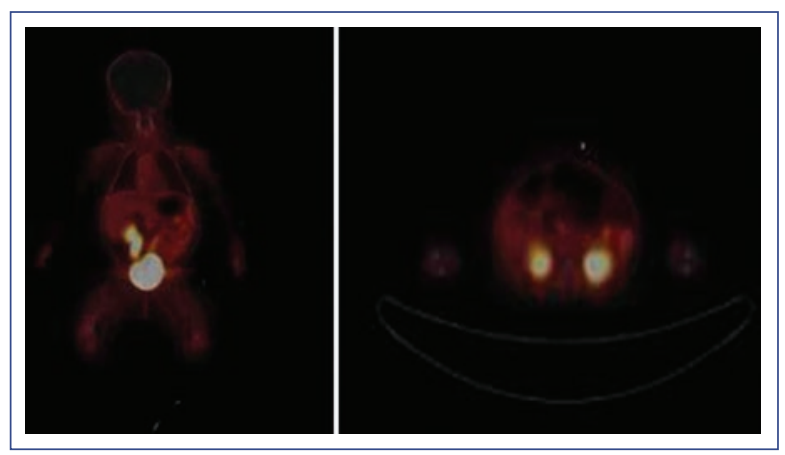

Figura 1. Estudio PET/TC con galio-68-dotatate realizado bajo anestesia general. Setenta minutos después de la inyección intravenosa del radiofármaco se adquirieron imágenes de cuerpo entero en una cámara Siemens-Biograph. Se realizó una TC para la corrección de atenuación y localización anatómica. Se identificó actividad focal con sobreexpresión de receptores de somatostatina en la región distal de la cola del páncreas.

presentaba consanguinidad familiar. En dos pacientes se detectó cardiomiopatía hipertrófica, aunque en ninguno se reportó antecedente de diabetes gestacional.

Los síntomas de HC se presentaron el primer día de vida en cinco casos, a los 5 días en un caso y a los 18 días de vida en el otro. Según su frecuencia, los síntomas más comunes fueron cianosis, hipotonía, convulsiones, dificultad respiratoria, apnea, bradicardia y somnolencia. Solo en una de las pacientes se detectó hipoglucemia sin haber presentado síntomas, ya que estuvo hospitalizada desde su nacimiento en la unidad de cuidados intensivos neonatales por un síndrome de dificultad respiratoria.

Aunque en todos los pacientes se realizó una prueba terapéutica con diazóxido, solo dos de ellos respondieron a este fármaco: uno a los 15 días después de su inicio, con una dosis de $20 \mathrm{mg} / \mathrm{kg} / \mathrm{día}$, y el otro a los 7 días de haber iniciado una dosis de $9 \mathrm{mg} / \mathrm{kg} / \mathrm{día}$. Los demás pacientes requirieron manejo con octreotida en incrementos progresivos. Dos pacientes respondieron a esta terapia: uno a la cuarta semana de tratamiento con $38 \mu \mathrm{g} / \mathrm{kg} / \mathrm{d}$ ía y el otro a la séptima semana de tratamiento con $40 \mu \mathrm{g} / \mathrm{kg} / \mathrm{dí}$. Los tres pacientes que no respondieron al manejo farmacológico fueron llevados a cirugía subtotal a los 3 meses de vida. De ellos, dos requirieron octreotida para su egreso, a dosis de 5 y $15 \mu \mathrm{g} / \mathrm{kg} / \mathrm{día}$, respectivamente. Solo uno de estos tres pacientes contó con estudio de tomografía por emisión de positrones (PET/TC) realizada con galio-68-dotatate. En este estudio se localizó una lesión con SUVmx: 1.94 (standardized uptake value ratio). Posteriormente, se realizó una resección dirigida a la cola pancreática (Figura 1). 
Tabla 2. Características de los pacientes con hiperinsulinismo congénito

\begin{tabular}{|c|c|c|c|c|c|c|c|}
\hline & \multicolumn{7}{|c|}{ Caso } \\
\hline Variable & 1 & 2 & 3 & 4 & 5 & 6 & 7 \\
\hline Sexo & $\mathrm{F}$ & $\mathrm{F}$ & M & M & M & $\mathrm{F}$ & $\mathrm{F}$ \\
\hline Consanguinidad & Sí & No & No & No & No & No & No \\
\hline Diabetes familiar & No & No & Sí & No & Sí & No & No \\
\hline $\begin{array}{l}\text { Cardiomiopatía } \\
\text { hipertrófica }\end{array}$ & No & No & Sí & Sí & No & No & No \\
\hline Pretérmino & No & Sí & No & No & No & No & No \\
\hline APGAR & $6 / 9 / 10$ & $8 / 9 / 10$ & $8 / 9 / 10$ & $6 / 8 / 9$ & $8 / 9 / 10$ & $8 / 9 / 10$ & $8 / 9 / 10$ \\
\hline $\begin{array}{l}\text { Insulina plasmática: } \\
\text { muestra crítica }(\mu \mathrm{U} / \mathrm{ml})\end{array}$ & 12.7 & 60.3 & 40.6 & 36.5 & 57.8 & 21.1 & 21.9 \\
\hline $\begin{array}{l}\text { Glucosa plasmática: } \\
\text { muestra crítica (mg/dl) }\end{array}$ & 27 & 19 & 13 & 34 & 33 & 41 & 28 \\
\hline Peso al nacimiento $(\mathrm{kg})$ & 3.3 & 3.7 & 3.7 & 5.0 & 4.1 & 2.9 & 3.8 \\
\hline $\begin{array}{l}\text { Inicio de síntomas } \\
\text { (días de vida) }\end{array}$ & 5 & 1 & 18 & 1 & 1 & 1 & 1 \\
\hline $\begin{array}{l}\text { Descripción de } \\
\text { síntomas }\end{array}$ & Asintomático & $\begin{array}{l}\text { Cianosis } \\
\text { Hipotonía }\end{array}$ & $\begin{array}{l}\text { Cianosis } \\
\text { Hipotonía } \\
\text { Disnea }\end{array}$ & $\begin{array}{l}\text { Cianosis } \\
\text { Hipotonía }\end{array}$ & $\begin{array}{c}\text { Apnea } \\
\text { Bradicardia } \\
\text { Convulsiones }\end{array}$ & $\begin{array}{l}\text { Somnolencia } \\
\text { Cianosis } \\
\text { Hipotonía }\end{array}$ & Apnea \\
\hline $\begin{array}{l}\text { Eventos secundarios } \\
\text { del diazóxido }\end{array}$ & Ninguno & Ninguno & Hipertricosis & Ninguno & Hipertricosis & Ninguno & Hipertricosis \\
\hline $\begin{array}{l}\text { Eventos secundarios } \\
\text { de la octreotida }\end{array}$ & Colelitiasis & - & Colelitiasis & Hipotiroidismo & Ninguno & - & Ninguno \\
\hline Tratamiento al egreso & $\begin{array}{c}\text { Octreotida } \\
40 \mu \mathrm{g} / \mathrm{kg} / \mathrm{día}\end{array}$ & $\begin{array}{l}\text { Diazóxido } \\
20 \text { mg/kg/ } \\
\text { día }\end{array}$ & $\begin{array}{l}\text { Octreotida } \\
5 \mu \mathrm{g} / \mathrm{kg} / \mathrm{dí} a\end{array}$ & $\begin{array}{l}\text { Octreotida } \\
15 \mu \mathrm{g} / \mathrm{kg} / \mathrm{día}\end{array}$ & $\begin{array}{c}\text { Octreotida } \\
38 \mu \mathrm{g} / \mathrm{kg} / \mathrm{día}\end{array}$ & $\begin{array}{l}\text { Diazóxido } \\
9 \mathrm{mg} / \mathrm{kg} / \mathrm{día}\end{array}$ & Ninguno \\
\hline Cirugía & No & No & Sí & Sí & No & No & Sí \\
\hline
\end{tabular}

F: femenino; M: masculino; APGAR: prueba de apariencia, pulso, gesticulación, actividad y respiración.

Dadas las características del sistema de salud colombiano, no fue posible el seguimiento ambulatorio de la mayoría de los pacientes. Sin embargo, sí fue posible la evaluación periódica del caso en que se realizó la resección quirúrgica dirigida con los resultados de la PET/TC. La última valoración se hizo 16 meses después de dicha resección. La paciente no requirió medicación adicional y permanece normoglucémica y sin hallazgos de retardo en el desarrollo psicomotor.

\section{Discusión}

Las urgencias de la hipoglucemia se manejan con la administración de dextrosa intravenosa, para asegurar la normoglucemia ${ }^{2}$. El flujo de glucosa que se administra debe ser similar al fisiológico producido por el hígado (8-10 $\mathrm{mg} / \mathrm{kg} / \mathrm{min}$ para neonatos o lactantes menores y $5-7 \mathrm{mg} / \mathrm{kg} / \mathrm{min}$ para niños) ${ }^{\dagger}$. En caso de HC, esta cantidad de glucosa es insuficiente. Se requieren flujos metabólicos de hasta $30 \mathrm{mg} / \mathrm{kg} / \mathrm{min}$, adicionales al manejo farmacológico, como con diazóxido y análogos de la somatostatina (octreotida). El glucagón (a dosis de $0.03 \mathrm{mg} / \mathrm{kg}$ intramuscular o subcutáneo, sin pasar de $1 \mathrm{mg}$ al día $\left.{ }^{1-4,6}\right)$ se usa para estabilizar a los pacientes en caso de pérdida de acceso venoso o hasta decidir el siguiente paso en el planteamiento terapéutico.

El diazóxido es un fármaco de primera línea, agonista del canal de potasio dependiente de ATP. Por esta razón, los pacientes con formas recesivas focales o difusas no responden bien a este tratamiento. En general, los pacientes que presentan alguna alteración en la enzima glutamato deshidrogenasa o en la 3-hidroxiacil-CoA-deshidrogenasa de cadena corta responden adecuadamente al diazóxido. Por otro lado, los pacientes con alteraciones 
relacionadas con la glucocinasa muestran una respuesta variable. El diazóxido se utiliza en dosis de 10-20 mg/kg/día, con aumentos progresivos para evaluar su efectividad. Sus principales reacciones adversas son la retención hídrica a corto plazo - para lo cual se recomienda la asociación con hidroclorotiazida- y la hipertricosis9.

Los análogos de la somatostatina, como la octreotida, se han usado de manera efectiva, ya que inhiben la secreción de insulina de forma distal a los canales de potasio, induciendo la hiperpolarización de las células beta e inhibiendo directamente los canales dependientes de calcio. Se pueden administrar por vía intravenosa o subcutánea (5-40 $\mu \mathrm{g} / \mathrm{kg} / \mathrm{día})$. Como efectos adversos relevantes de este grupo de fármacos se encuentran la taquifilaxia después de algunas dosis, así como la disminución en la motilidad de la vesícula biliar y en la secreción de bilis, que puede producir esteatorrea, colestasis, disfunción hepática y colelitiasis ${ }^{5}$. También puede presentarse enterocolitis, la cual no se identificó en la serie de casos descrita en este trabajo.

En los pacientes en quienes el tratamiento farmacológico no logra la normoglucemia es necesario proceder a manejo quirúrgico. Es fundamental establecer si el paciente cursa con una forma difusa o focal de la enfermedad, puesto que en aquellos con hiperinsulinismo congénito focal, la resección sería delimitada al área de la lesión, mientras que en aquellos con hiperinsulinismo congénito difuso, el manejo sería la pancreatectomía total ${ }^{1,5,7}$. Para determinar el tipo de HC, el estudio de elección es la PET/TC, usando como radiotrazador 18 fluoro-dihidroxifenilalanina decarboxilasa (18-fluoro-L-dopa), que cuenta con una sensibilidad reportada del $85-100 \%$, una especificidad del $96 \%$ y un valor predictivo positivo del $96 \%$. Algunos autores han propuesto el uso de una tasa de valor de captación estandarizada (SUVr) > 1.2 y 1.5 como criterio diagnóstico, pero otros no lo recomiendan por la buena correlación existente entre el análisis visual del estudio imagenológico y la localización de la lesión focal $^{11-13}$. Las imágenes con galio-68-dotatate como radiotrazador se han utilizado en adultos para la localización de insulinomas y tumores neuroendocrinos, con una sensibilidad reportada del $25.8 \%$ y una especificidad del $25 \%$; incluso, existen algunos reportes de éxito en la localización de la lesión en niños con hiperinsulinismo ${ }^{11,14,15}$.

El HC es una condición clínica compleja que amerita un diagnóstico preciso y un manejo adecuado, con el objetivo de evitar el daño neurológico que producen las hipoglucemias persistentes y proporcionar la mejor opción terapéutica disponible.

Los pacientes que respondieron a diazóxido $(n=2)$ y los que respondieron a octreotida $(n=2)$ egresaron de la institución con normoglucemia, continuaron con el mismo tratamiento y presentaron una adecuada respuesta clínica. Los pacientes que no respondieron al tratamiento farmacológico fueron sometidos a una pancreatectomía subtotal del $97 \%$, y dos de ellos requirieron adicionalmente octreotida a bajas dosis.

En Colombia, a pesar de realizarse la PET/TC, el 18-fluoro-L-dopa no está disponible como radiotrazador para localizar la lesión en caso de requerir manejo quirúrgico. Sin embargo, se contaba con otro radiotrazador (galio-68-dotatate) al momento del diagnóstico del último de los pacientes descritos, lo cual permitió encontrar una lesión focal en la cola del páncreas. Con este hallazgo, se realizó una resección limitada de dicha lesión. El paciente egresó con normoglucemia, sin otro tratamiento. Al disponer de este estudio, se logró evitar riesgos asociados con la resección subtotal, como el desarrollo de diabetes mellitus y de insuficiencia pancreática exocrina. Este último paciente es el primer caso descrito en Colombia en que se utilizó galio-68-dotatate como radiotrazador alternativo.

\section{Responsabilidades éticas}

Protección de personas y animales. Los autores declaran que para esta investigación no se han realizado experimentos en seres humanos ni en animales.

Confidencialidad de los datos. Los autores declaran que han seguido los protocolos de su centro de trabajo sobre la publicación de datos de pacientes.

Derecho a la privacidad y consentimiento informado. Los autores han obtenido el consentimiento informado de los pacientes o sujetos referidos en el artículo. Este documento obra en poder del autor de correspondencia.

\section{Conflicto de intereses}

Los autores declaran no tener ningún conflicto de intereses.

\section{Financiamiento}

Ninguno.

\section{Agradecimientos}

A la Dra. Mónica Cediel, por su participación activa como pediatra tratante en el cuidado y manejo de varios de los pacientes incluidos en esta serie de casos. Al Departamento de Educación e Investigación del 
Instituto Roosevelt, por su ayuda y colaboración en este manuscrito.

\section{Bibliografía}

1. Arnoux JB, de Lonlay P, Ribeiro MJ, Hussain K, Blankenstein O, Mohnike K, et al. Congenital hyperinsulinism. Early Hum Dev. 2010;86:287-94.

2. Lord K, Dzata E, Snider KE, Gallagher PR, De León DD. Clinical presentation and management of children with diffuse and focal hyperinsulinism: a review of 223 cases. J Clin Endocrinol Metab. 2013;98:E1786-9.

3. Hernández MI, Hodgson MI, Cattani A. Hiperinsulinismo neonatal persistente: análisis del diagnóstico diferencial a propósito de dos casos clínicos. Rev Med Chile. 2004;132:995-1000.

4. Petraitienè I, Barauskas G, Gulbinas A, Malcius D, Hussain K, Verkauskas G, et al. Congenital hyperinsulinism. Medicina (Kaunas). 2014;50:190-5.

5. Hussain K. Congenital hyperinsulinism. Semin Fetal Neonatal Med. 2005;10:369-76.

6. Meissner T, Mayatepek E. Clinical and genetic heterogeneity in congenital hyperinsulinism. Eur J Pediatr. 2002;161:6-20.

7. Ismail D, Smith VV, de Lonlay P, Ribeiro MJ, Rahier J, Blankenstein O, et al. Familial focal congenital hyperinsulinism. J Clin Endocrinol Metab. 2011;96:24-8
8. Stanley CA. Hyperinsulinism in infants and children. Pediatr Clin North Am. 1997;44:363-74.

9. Palladino AA, Bennett MJ, Stanley CA. Hyperinsulinism in infancy and childhood: when an insulin level is not always enough. Clin Chem. 2008;54:256-63.

10. Stanley CA. Perspective on the genetics and diagnosis of congenital hyperinsulinism disorders. J Clin Endocrinol Metab. 2016;101:815-26.

11. Nockel $P$, Babic B, Millo $C$, Herscovitch $P$, Patel D, Nilubol N, et al. Localization of insulinoma using $68 \mathrm{Ga}$-dotatate PET/CT scan. J Clin Endocrinol Metab. 2017;102:195-9.

12. Ribeiro MJ, Boddaert N, Bellanné-Chantelot $C$, Bourgeois S, Valayannopoulos V, Delzescaux T, et al. The added value of [18F]fluoro-L-DOPA PET in the diagnosis of hyperinsulinism of infancy: a retrospective study involving 49 children. Eur J Nucl Med Mol Imaging. 2007; $34: 2120-8$.

13. Laje $P$, States LJ, Zhuang $H$, Becker SA, Palladino AA, Stanley CA, et al. Accuracy of PET/CT scan in the diagnosis of the focal form of congenital hyperinsulinism. J Pediatr Surg. 2013;48:388-93.

14. Arun S, Rai Mittal B, Shukla J, Bhattacharya A, Kumar P. Diffuse nesidioblastosis diagnosed on a Ga-68 dotatate positron emission tomography/computerized tomography. Indian J Nucl Med. 2013;28:163-4.

15. Canbaz F, Aydin M, Can Meydan B, Ceyhan Bilgici M, Aritürk E. Striking visualization of diffuse congenital nesidioblastosis on Ga-68 dotatate PET/CT. Mol Imaging Radionucl Ther. 2019;28:83-5. 